\title{
Analysis of Discrete Time Schemes for Milling Forces Control under Fractional Order Holds
}

\author{
Luis Rubio',\#, Manuel De la Sen², Andrew Peter Longstaff', and Alan Myers' \\ 1 Centre for Precision Technologies, University of Huddersfield, Queensgate, Huddersfield, United Kingdom, HD1 3DH \\ 2 Institute of Research and Development of Processes, University of the Basque Country, Barrio Sarriena s/n, Leioa, Bizkaia, Spain, 48940 \\ \# Corresponding Author / E-mail: L.R.Rodriguez@hud.ac.uk, TEL: +44-1484-471805, FAX: +44-1484-472161
}

KEYWORDS: Cutting forces, Milling, Model Reference Control, Fractional Order Holds, Multi-models

In this paper, discrete time model reference control schemes for practical milling using different discretization of the continuous-time plant are presented. First, a basic controller scheme is addressed where a fractional order hold with pre-fixed value of the gain is used. Secondly, a multi-model scheme, which outputs different discretization in parallel with the continuous-time milling system transfer function under a fractional order hold (FROH) of correcting $\beta \in[-1,1]$, is dealt with. Then, an intelligent design framework is designed as a supervisory scheme with two hierarchical levels in order to find the most appropriate value for the gain $\beta$. For choosing the value of $\beta$, a tracking performance index is designed. It evaluates each pre-defined discretization of the continuous time milling transfer function and the scheme chooses the one with the smallest value of the index in order to generate the real control input to the plant. Two different methods of adjusting this value are presented and discussed. The first one selects a $\beta$-value among a fixed pre-defined set of possible values, while the second one the value of $\beta$ is updated by adding or subtracting a small quantity.

Manuscript received: November 15, 2012 / Accepted: January 29, 2013

\section{NOMENCLATURE}

$a_{d c}=$ axial depth of cut $(\mathrm{mm})$

$f_{a}=$ actual feed velocity $(\mathrm{mm} / \mathrm{s})$

$f_{c}=$ command feed velocity $(\mathrm{mm} / \mathrm{s})$

$J_{c}=$ continuous response characterization

$J_{k}=$ discrete performance index

$F_{r}=$ reference force $(N)$

$F_{p}=$ resultant force $(N)$

$F_{p m}=$ model reference resultant force $(N)$

$n_{l}=$ number of parallel models/switching

$N_{t}=$ number of teeth

$r_{d c}=$ non-dimensional immersion function

$S_{s}=$ spindle speed $(\mathrm{rev} / \mathrm{s})$

$T=$ sampling time

$T_{r}=$ rise time $(s)$

$T_{s}=$ switching sampling times sequence

$\omega_{n}=$ natural frequency $(\mathrm{rad} / \mathrm{s})$

$\xi=$ damping ratio

$\tau_{s}=$ average time constant $(s)$

$\tau_{c}=$ cutting process time constant $(s)$

$\phi_{s t}=$ start angle (degrees)

$\phi_{e x}=$ exit angle (degrees)

\section{Introduction}

Milling, the cutting process widely used in the manufacturing of mechanical components, consists of the relative movement between clamped workpiece and rotating multi-tooth cutting tool. Malfunctions, such as tool wear or fracture, can appear during general working of the process. Other important and undesired effects to take into account are chatter vibrations. ${ }^{1,2}$ In order to decrease these malfunctions in the process the peak cutting force on the workpiece has to be maintained below a prescribed safety upper-bound. Some works have been developed in the identification of the milling forces. ${ }^{3}$ Furthermore, achieve certain degree of quality in the surface finish of the workpiece is required. ${ }^{4,5}$ Those facts imply that a control strategy has to be implemented on the milling system in order to fulfill such safety and performance requirements.

The problem of adaptively controlling milling forces has been widely addressed during last three decades in the manufacturing literature. Early works were presented by Koren and Masory ${ }^{6,7}$ and Tomizuka. ${ }^{8}$ The adaptive control was presented as an alternative to initial fixed gain controllers in order to stabilize the milling system and reduce large overshoot. Landerbaugh and Ulsoy, also, applied Model 
Reference Adaptive Controller (MRAC) to the milling process, ${ }^{9}$ which was originally presented by Tomizuka. ${ }^{8}$ MRAC controllers were physically implemented in. ${ }^{10}$ Other control schemes applied to milling problem are model based approaches, ${ }^{11}$ neural networks ${ }^{12}$ and fuzzy logic. ${ }^{13}$ Recently, model based adaptive control schemes are still widely applied to the milling system for controlling forces ${ }^{14}$ and for achieving better surface finish. ${ }^{15}$

To address the milling control problem, designers have traditionally used zero order holds $(\mathrm{ZOH})$. However, different kinds of holds are increasingly being used due to their enhanced properties. ${ }^{16,17}$ In this work, strategies for controlling the milling system are based on the use of fractional order holds of the correcting gain $\beta \in[-1,1],(\beta-F R O H)$. It shows the influence of the gain $\beta$ when using a fixed $\beta-F R O H$ in the closed loop behavior and, it investigates the performance of two different multi-model schemes. It is supported by the idea that $\beta-F R O H$ with intermediate adaptation rate modifies the location of the zeros of the discrete transfer function with respect to those obtained from the use of a zero-order-hold ( $\mathrm{ZOH})$ or a first-order-hold (FOH). Two possible advantages are: a) the stability degree of stable zeros can be improved so that some of them can be better cancelled when implementing a model-following control design while improving the transient closed-loop behavior; b) the discrete control can better accommodate the rippling effect in-between any two consecutive sampling instants when applying a discrete-time control to a continuous-time plant. The reason is that there is there is a kind of practical interpolation in continuous time from measured data inbetween two consecutive such samples due to the structure of the $\beta$ $F R O H$ what allows to accommodate the control law in continuous time while just using discrete-time data. ${ }^{18-20}$ As a result, the controller can lead, for example, to produce better surface finish and maintenance of the tool and machine tool components and to minimize wear of the tool while avoiding or minimizing electrical ripple. Multi-model schemes are also useful, for instance, when the system works in different states governed by different equations from the milling case or, to modify the closed-loop structure in one working point achieving best tracking performance. So, the algorithm methodologies can be useful when the holistic control of the milling process is taking into account in order to achieve better surface finish or to deal with some kind of intrinsic nonlinearity. Also, FROH has potential benefits when dealing with ramp form milled parts or to improve transient responses leading to further potential benefits.

Then, firstly, a basic model is introduced following a closed control loop. In this case, the continuous plant of the milling system is discretized under a fixed $\beta$-value of the $\beta$-FROH. Secondly, a multimodel control system obtains a series of discrete-time models of the system in parallel. Each different discretization of the plant has an associated controller. ${ }^{16,17}$ Also, logic based on how the controller works is incorporated and modified in real time. For this purpose, an intelligent design framework has been designed as supervisory scheme. It consists of a switching rule and a tracking performance index which evaluates each possible discretization. The scheme chooses the controller which is associated to the lowest value of the tracking performance index for implementing the $\beta-F R O H$ device and the control law.

Finally, the tracking performance of the continuous -time output signal is studied. It is carried out by means of a cost function and applied to the basic control scheme when just $\mathrm{ZOH}$ and $\beta-F R O H$ fixed gain devices and when the two different structures of the multi-model scheme are applied.

The work is organized as follows. In the next section the continuous model and its discretization under a $\beta-F R O H$ is presented and the model reference transfer function is displayed. In the third section, basic model based reference control scheme with fixed gain of the $\beta$ $F R O H$ and, two different parameterizations of model reference control schemes based on multiple discretization of the continuous plant using a $\beta-F R O H$ are addressed. Section four introduces a switching rule and identification performance index, followed by the tracking performance of the continuous-time output, in section five. Finally, simulation results are covered in the section six, ending the paper with a discussion and some conclusions.

In practice, batch and high volume milling environments are characterized by fixing, or varying with a known range, the tool-part combinations providing a predictable solution. Meanwhile, job-shop environments require adaptive techniques since tool-part combinations are different at each operation. In this paper, the design of a discrete time control of milling forces is presented considering high volume operations, where the milling system model is perfectly characterized and the plant parameters are known or varied in a known way, even though sudden changes in the tool-part combinations happen.

\section{System description}

\subsection{Continuous model}

The milling system can be modelled as the series decomposition of a Computerized Numerical Control (CNC), which includes all the circuitry involving in the workpiece feed movement (amplifiers, motor drives), and the tool-work-piece interaction model itself. A feed rate command $f_{c}$ (which plays the role of the control signal) is sent to the $\mathrm{CNC}$ unit. This feed rate represents the desired velocity for the relative tool-to-workpiece movement. Then, the $\mathrm{CNC}$ unit manages to make the feed movement at an actual feed velocity of $f_{a}$ according to the CNC dynamics. Even though the machine tool drive servos are typically modelled as high order transfer functions, they can usually be approximated as a second order transfer function within the range of working frequencies. Since, they are tuned to be over-damped without overshoot, so that they can be modelled as the first order system of transfer function: ${ }^{10}$

$$
G_{s}(s)=\frac{f_{a}(s)}{f_{c}(s)}=\frac{1}{\tau_{s} s+1}
$$

where $f_{a}$ and $f_{c}$ are the actual and command velocity values of the table in $\mathrm{mm} / \mathrm{s}$ respectively and $\tau_{\mathrm{s}}$ is an average time constant, which depends on the type of the machine tool.

In addition, the chatter vibration and resonant free cutting process can be approximated as the first order system: ${ }^{10}$

$$
G_{p}(s)=\frac{F_{p}(s)}{f_{a}(s)}=\frac{K_{c} a_{d c} r\left(\varnothing_{s t}, \varnothing_{e x}, N_{t}\right)}{N_{t} S_{s}} \frac{1}{\tau_{s} s+1}
$$

where $F_{p}$ is the resultant cutting force $(N), K_{\mathrm{c}}\left(\mathrm{N} / \mathrm{mm}^{2}\right)$ is the cutting 
pressure constant, $a_{d c}(m m)$ is the axial depth of cut, $r_{d c}\left(\phi_{s t}, \phi_{e x}, N_{t}\right)$ is a non-dimensional immersion function, ranging between 0 and $\left(N_{t}\right)$ depending on the immersion angle and the number of teeth in cut, is the number of teeth on the milling cutter, $S_{s}(\mathrm{rev} / \mathrm{s})$ is the spindle speed and $\tau_{\mathrm{c}}$ is a cutting process constant parameter.

The combined transfer function of the system obtained from (1) and (2) is

$$
G_{c}(s)=\frac{F_{p}(s)}{f_{c}(s)}=\frac{K_{p}}{\left(\tau_{c} s+1\right)\left(\tau_{s}+1\right)}
$$

where the process gain is $K_{p}(k N \cdot s / m m)=K_{c} a_{d c} r_{d c} / N_{t} S_{s}$.

\subsection{Discrete model under $\beta$-FROH}

In this paper, the problem of controlling the milling continuous plant is addressed by using a discrete controller. The discrete controller is obtained by applying a model reference pole placement based control design to a discrete model of the plant (3) obtained by means of a FROH with a certain correcting gain, $\beta$. The additional "degree of freedom" $\beta$ provided by the $\beta-F R O H$ can be used with a broad variety of objectives such as to improve the transient response behavior ${ }^{16-18}$ and the stability properties of the zeros of the discretized systems ${ }^{16-20}$ or, to enhance inter-sample behavior or avoid ripple between sampling times. ${ }^{16,19,20}$ Hence, the discretization of the continuous transfer function of (3) under a $\beta-F R O H$ is calculated as:

$$
H_{\beta}(z)=Z\left[h_{\beta}(s) \cdot G_{c}(s)\right]
$$

$$
\text { where } h_{\beta}(s)=\left(1-\beta e^{-s T}+\frac{\beta\left(1-e^{-s T}\right)}{T s}\right) \frac{1-e^{-s T}}{T s}
$$

is the transfer function of a $\beta-F R O H$, where $\mathrm{z}$ is the argument of the $Z$-transform, being formally equivalent to the one step ahead operator, $q$, used in the time domain representation of difference equations. This allows us to keep a simple unambiguous notation for the whole paper content. The sampling time $T$ has been chosen to be inversely proportional to the spindle speed, $S_{s}$, as it is usual for this kind of systems. ${ }^{10}$ Furthermore, $\mathrm{H}_{\beta}(z)$ may be calculated using just $\mathrm{ZOH}$ devices in the following way:

$$
\begin{aligned}
& H_{\beta}(z)=\frac{B_{\beta}(z)}{z^{\delta_{\beta}} A(z)}=\frac{z-\beta}{z} Z\left[h_{o}(s) G_{c}(s)\right]+\frac{\beta(z-1)}{T z} Z\left[h_{o}(s) \frac{G_{c}(s)}{s}\right]= \\
& =\frac{B_{\beta}(z)}{z^{\delta_{\beta}}\left(z-e^{T / \tau_{m}}\right)\left(z-e^{T / \tau_{c}}\right)}
\end{aligned}
$$

where $h_{o}(s)=\frac{1-e^{-s T}}{s}$ is the transfer function of a $Z O H$ and $\delta_{\beta}=1$ if $\beta \neq 0$ and $\delta_{\beta}=0$ if $\beta=0$, which means that a fractional order hold with $\beta \neq 0$ adds a pole at the origin.

\subsection{Desired response: model reference}

A second order system,

$$
G_{m}(s)=\frac{\omega_{n}^{2}}{s^{2}+2 \xi \omega_{n} s+\omega_{n}^{2}}
$$

is selected to represent the system model reference. This system is characterized by the desired damping ratio, $\xi$, and it's natural frequency, $\omega_{n}$. It is known that small $\xi$ leads to a large overshoot and large setting time. A general accepted range value for $\xi$ to attain satisfactory performance is between 0.5 and 1 , which corresponds to the so-called under-damped systems. In this way, a damping ratio of $\xi=0.7$ and a rise time, $T_{r}$, equal to three spindle periods is usually selected for practical applications. Furthermore, the natural frequency is then usually suggested to be $\omega_{n}=2.5 / T_{\mathrm{r}} \mathrm{rad} / \mathrm{s}$. This continuous time model reference is then discretized with the same $\beta-F R O H$ as the real system was in order to obtain the corresponding discrete-time reference model for the controller. Thus, a number of different discrete models obtained from a unique continuous reference model are considered depending on the value of $\beta$ used to obtain the discretization.

\section{Control schemes}

\subsection{Basic model following scheme}

The aim of the model following control strategy is to force the closed-loop system to behave as a prescribed reference model. Thus, the model following control scheme depicted in figure 1 is applied. In the scheme, $H_{f f}(z)=\frac{T(z)}{R(z)}$ is the feed-forward compensator, $H_{f c}(z)=\frac{S(z)}{R(z)}$ is the feedback compensator, $H_{\beta}(z)$ is the discrete plant, $H_{m, \beta}(z)$ is the discrete-time reference model and $F_{r, k}$ is the reference force.

The transfer function of the reference model is,

$$
H_{m}(z)=\frac{B_{m}(z)}{A_{m}(z)}=\frac{B_{m}(z) A_{o}(z)}{A_{m}(z) A_{o}(z)}=\frac{B^{-}(z) B_{m}^{\prime}(z) A_{o}(z)}{A_{m}(z) A_{o}(z)}
$$

where $B_{m}^{\prime}(z)$ contains the free-design reference model zeros, $B^{-}(z)$ is formed by the transmitted unstable (assumed known) plant zeros and $A_{o}(z)$ is a polynomial including the eventual closed-loop stable polezero cancelations. Those cancellations are normally introduced when it is necessary to guarantee that the relative degree of the reference model will be no less than that of the closed-loop system, so that the synthesized controller will be causal. Then, the polynomials $R(z), S(z)$ and $T(z)$ have to be synthesized ( $T(z)$ depends only on the reference model zeros polynomial which is of constant coefficients) where

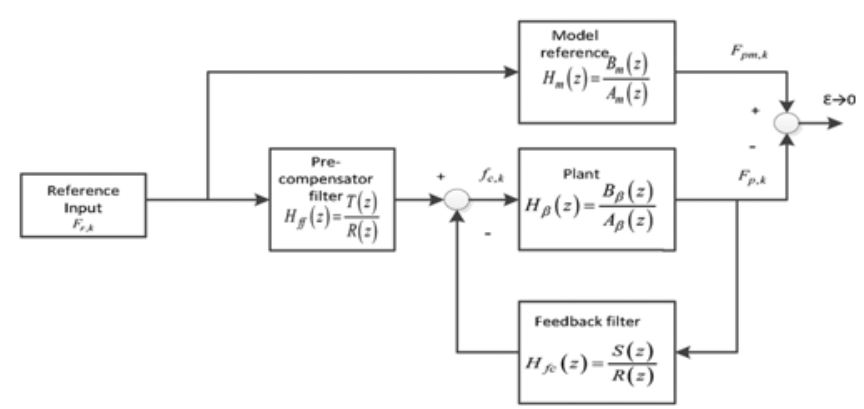

Fig. 1 Basic model following scheme diagram 
$T(z)=B^{\prime}{ }_{m}(z) A_{o}(z), R(z)$ (monic) and $S(z)$ are unique solutions with degrees fulfilling

$$
\operatorname{deg}(\mathrm{R})=n, \operatorname{deg}(S)=n-1, \operatorname{deg}\left(A_{m} A_{o}\right)=2 n
$$

of the polynomial Diophantine equation

$$
A(z) R(z)+B(z) S(z)=B^{+}(z) A_{m}(z) A_{o}(z) \leftrightarrow A(z) R_{1}(z)+B^{-}(z) S(z)=A_{m}(z) A_{o}(z)
$$

with $R(z)=B(z) R_{1}(z)$ at every sampling instant. Note that, $n=2$ when a $\mathrm{ZOH}$ is used in view of the second order milling plant and reference continuous time model transfer function. For a $\beta-F R O H$ not being $Z O H, n=3$ since new pole at the origin is automatically added to the plant.

From (8)-(9), perfect tracking is achieved through the control signal:

$$
f_{c, k}=\frac{T(z)}{R(z)} F_{r, k}-\frac{S(z)}{R(z)} F_{p, k}
$$

\subsection{Multi-model schemes}

Two alternative organizations of the set of discrete models are proposed and discussed in this sub-section of the paper.

\subsubsection{Multi-model scheme 1}

The first architecture proposes that the transfer function composing the parallel scheme is obtained from pre-intended values of $\beta$. Thus, a finite set of possible design values is considered, obtaining the set of transfer functions as,

$$
H_{\beta}(z)=Z\left[h_{\beta}(s) G(s)\right], \beta \in\left\{\beta^{(1)}, \beta^{(2)}, \ldots, \beta^{\left(n_{m}\right)}\right\}
$$

Then, the actual control law at the previous time instant is applied to the above scheme by calculating the output of all continuous time models $F_{p 1}(t), F_{p 2}(t), \ldots, F_{p n_{m}}(t)$. The tracking performance of the set of possible models is evaluated by comparing these outputs and desired reference output. Finally, the proposed high level supervision algorithm will select the most appropriate one to design the control law which is actually applied to the system according to the values of these performance indexes. It is explained in the next sub-section. Figure 2 depicts a schematic representation of the control scheme.

\subsubsection{Switching rule and identification performance index}

The objective of the supervisor is to evaluate the tracking

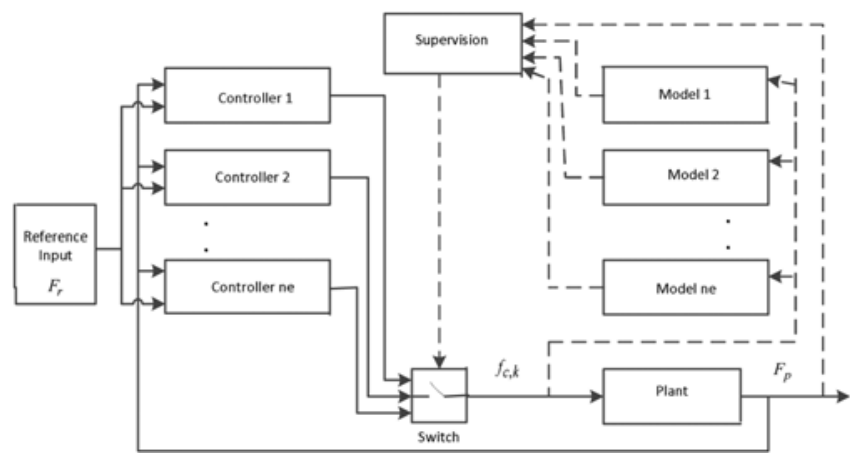

Fig. 2 Multi-model scheme of the model following control loop performance of the possible controllers operating on the plant for the given reference model with the aim of choosing the current controller from the set of parallel controllers. In this section the switching rule and identification performance index, which are implemented in the closed loop of the milling system controller, are explained.

\subsubsection{Switching rule}

The switching rule for the model reference control reparameterization is obtained from the performance index $J_{k}^{(l)}$, presented in next subsection, as follows:

1. Let the switching sampling time sequence be denoted by $T_{s}=\left\{t^{(1)}, t^{(2)}, \ldots, t^{\left(n_{1}\right)}\right\}$ where $n_{l}$ is the number of switchings and $\left(t^{(i+1)}-t^{(i)}\right) \geq \tau_{R}=N_{r} T, N_{r} \in N$ where $T$ is the sampling time, a known minimum residence time (or if it is unknown, then, it is replaced by some available upper-bound), for all $t^{(i)}, t^{(i+1)} \in T_{s}$. The minimum residence time is given by the necessary time to ensure the stability of the closed-loop system. During this period of time, it is not allowed to switch to other parallel controller in order to guaranty the stability of the closed loop system. Then, the chosen controller to generate the control signal to the plant is fixed during $N_{r}$ times the sampling time.

2. Thus, the $c_{k}$-model scheme with $1 \leq c_{k} \leq n_{l}$, which parameterize for all $k>0$ the basic controller at any switching time, , is updated as follows. Assume that the last switching time for the controller reparameterization was $t^{(i)}$. Thus, for each current $k^{\text {th }}$ sampling time, define the auxiliary integer variable:

$\overline{c_{k}}=\arg \left[i: J_{k}^{(i)}=\min \left(J_{k}^{(i)}\right) ; i, j \in n_{l}\right]$, for all integer $k \geq 1$,

1. if $k T \geq t^{(i)}+\tau_{R}$ then $c_{k} \leftarrow \overline{c_{k}}$.

2. if $c_{k} \neq c_{k+1}$ then $t^{(\mathrm{i}+1)} \leftarrow k T$ and modify $T_{s} \leftarrow\left\{T_{s}, t^{(\mathrm{i}+1)}\right\}$.

Note that it does not necessary means that the update of the $\beta$-value has to be at each switching instant.

The value of the minimum residence time which ensures the closed loop stability could be obtained from the parameters of the system ${ }^{21}$ or from 'a priori' knowledge through an algorithm. ${ }^{22}$ However, it is know that there exists a minimum value of the residence time which guaranties the stability of the closed-loop system. ${ }^{18,22}$ This can be mathematically expressed as:

1. For each $k \geq k_{1}$, if $\min \left(\sum_{i=k-k_{j}}^{k} f_{j}^{(i)}\right) \geq \overline{J_{M}}, 1 \leq i, j \leq n_{e}$ then $\tau_{R} \leftarrow \tau_{R}+\Delta \tau_{R 1}$ until closed loop stability is guaranteed by an admissible range $\tau_{R} \in\left(\underline{\tau_{R}}, \infty\right)$.

2. But,

$$
\begin{aligned}
& \max \left(\sum_{j=k-k_{1}}^{k}\left|J_{j}^{(i)}-J_{j}^{\left(c_{k}\right) \mid}\right|\right) \leq \underline{J_{M}} \& \tau_{R} \in\left(\underline{\tau_{R}}, \infty\right), 1 \leq i \leq n_{e} \\
& \text { then } \tau_{R} \leftarrow \tau_{R}-\Delta \tau_{R 2} \text { for each } k \geq k_{2} .
\end{aligned}
$$

being $\overline{J_{M}}$ and $J_{M}$ prefixed real positive constants, sufficiently large and small respectively and, the initial value of $\tau_{R}$ arbitrary. 
The parameters $\overline{J_{M}}, J_{M}, \Delta \tau_{R 1}, \Delta \tau_{R 2}$ are supposed to be programmed by the designer according to previous knowledge and insight of the problem at hand. $k_{1}$ and $k_{2}$ are integers. This part of the algorithm has to be run on-line or off-line during a short period of time being significantly less than the relevant transient response duration.

\subsubsection{Identification performance index}

A general performance index is now proposed which is able to evaluate the performance of the closed loop $\left(F_{p m}^{(l)}-F_{p}^{(l)}\right)$, the jump in the output associates to a switching between controls $\left(F_{p, k}^{\left(c_{k-1}\right)}-F_{p, k}^{(l)}\right)$, the cost of the control $\left(F_{c, k}^{\left(c_{k-1}\right)}-F_{c, k}^{(l)}\right)$ and the output signal error $\left(F_{p}-F_{p}^{(l)}\right)$. Then, the following cost function is suggested:

$$
\begin{aligned}
& J_{k}^{(l)}=\alpha_{k} \sum_{j=k-M}^{k} \lambda^{k-j}\left(F_{p m}^{(l)}-F_{p}^{(l)}\right)^{2}+\beta_{k}\left(F_{p, k}^{\left(c_{k-1}\right)}-F_{p, k}^{(l)}\right)^{2}+ \\
& +\gamma_{k}\left(F_{c, k}^{\left(c_{k-1}\right)}-F_{c, k}^{(l)}\right)^{2}+\delta_{k} \sum_{j=k-M}^{k} \lambda^{k-j}\left(F_{p}-F_{p}^{(l)}\right)^{2}
\end{aligned}
$$

for $1 \leq l \leq n_{l}$, where $\lambda \in[0,1]$ and $M>0$ are designed as real parameters, being $M$ big enough to evaluate the performance of the system. $\lambda$ and $\lambda^{\prime}$ are forgetting factors which allow to give more importance to the last time interval values, $\alpha_{k}+\beta_{k}+\delta_{k}+\gamma_{k}=1, \alpha_{k}, \beta_{k}, \delta_{k}, \gamma_{k}>0$, and $F_{p m}^{(l)}$ represents the force output of the reference model in the l-parallel control, $F_{p m}^{(l)}$ the output of the plant, $F_{p, k}^{\left(c_{k-1}\right)}$ the output associated to the $c_{k-1}$ model in the $k$-sample, $f_{p, k}^{(l)}$ the output associated to the $l$ model at the $k$ sample, $f_{c, k}^{(l)}$ the control signal associated to the $l$ model in the $k$ sample, $F_{p}$ the control signal associated to the $l$ model at the $k$-sample, $F_{p}$ the output of the system and $F_{p}^{(l)}$ the output of the system associated to the $l$ model.

\subsubsection{Multi-model scheme 2}

In the second criterion, the system starts with an arbitrary value and its associate tracking performance is compared with that obtained from the use of other two closes values of $\beta$, one of which is slightly larger and the other slightly smaller than the active value. Then, the system only can choose a value among those three cases. However, these three potential values are not constant in general and; they are updated in the case of choosing one of the two other close values to the current value of $\beta$. Then, it becomes the active one and the other two are updated again by adding and subtracting a quantity. If the system chooses to maintain the same value of $\beta$, then the other two possible values are updated as well considering other two closer values of $\beta$.

The following algorithm describes carefully this method:

1. At $k^{\text {th }}$ sample the active value of $\beta$ is $\beta_{k}$. Another two values, $\beta_{k}^{\text {sup }}=\beta_{k}+\Delta \beta_{k}$ and $\beta_{k}^{\text {inf }}=\beta_{k}-\Delta \beta_{k}$ are used for simulation purposes, supposing that the last switching of $\beta$ took place at the $\bar{k}^{\text {th }}$ sample.

2. If $(k+1) T-\bar{k} T \geq M T, M>0$, then the values of the tracking performance of the three possible discretizations are compared and the one with the lowest value of the switching is used in the $\beta$ FROH device.

3. If the system chooses to maintain the same value of $\beta$, then first,
$\Delta \beta_{k}$ is decreased and then $\beta^{\text {sup }}$ and $\beta^{\text {inf }}$ are updated. Mathematically expressed as $\beta_{k+1}=\beta_{k}, \Delta \beta_{k}=\Delta \beta_{k} / m, m>1$ and $\beta_{k+1}^{\text {sup }}=\beta_{k}+\Delta \beta_{k}, \beta_{k+1}^{\text {inf }}=\beta_{k}-\Delta \beta_{k}$.

4. If the system chooses another value, $\Delta \beta_{k}$ maintains its value and $\beta^{\text {sup }}$ and $\beta^{\text {inf }}$ are calculated by adding and subtracting the following value:

$$
\begin{aligned}
& \text { - If } \beta_{k+1}=\beta_{k}^{\text {sup }}, \beta_{k+1}^{\text {sup }}=\beta_{k}+2 \Delta \beta_{k}, \beta_{k+1}^{\text {inf }}=\beta_{k} . \\
& \text { - If } \beta_{k+1}=\beta_{k}^{\text {inf }}, \beta_{k+1}^{\text {sup }}=\beta_{k}, \beta_{k+1}^{\text {inf }}=\beta_{k}-2 \Delta \beta_{k} .
\end{aligned}
$$

The reasons to propose this second multi-model scheme is to avoid bad transient in switching instants due to large changes in the gain of the $\beta$-FROH.

Note that there are two supervisory hierarchical levels of action of the intelligent framework:

1. Basic control: It consists of generating the control signal from (10) for each discrete model integrated in the multi-model scheme.

2. Choice of $\beta$ : The model and gain of the $\beta$-FROH is selected on-line via minimization of the supervisory performance index (13) and the criterion selected for choosing $\beta$.

In summary, the output of the closed loop of each controller using different $\beta$ 's is stable. It is intended to improve the performance of the system using multiple models and switching. The drawback of switching is that it can destabilize the system if it is done badly. The source of instability is that switching generates transitory responses and, if it is switched to a new parameterization when the input is still transitory, the superposition of the transitory states could lead to an unstable system. To preserve stability it is necessary to respect a residence time. It is interesting to determine the residence time in order to minimize its value while guaranteeing stability.

\section{Continuous response characterization}

In this section, the output of the milling system is evaluated under the application of the developed control schemes using fractional order holds. It is compared with the output of the basic control scheme represented in section 3 under a $\mathrm{ZOH}$ discretization. Then, a cost function is introduced in this section in order to characterize the continuous response of the system. This cost function measures the area which is formed between the output force continuous response and the model reference continuous response. It is defined as:

$$
J_{c}=\sum_{j=1}^{k_{n}} \int_{(j-1) T}^{j T}\left(F_{p}(\tau)-F_{p m}(\tau)\right)^{2} d \tau
$$

where $F_{p}(t)$ is the continuous time force output of the system and $F_{p m}(t)$ is the continuous time of the model reference force output.

The continuous time response of the force output is calculated as the output of the continuous transfer function using the continuous feed rate as input. The continuous feed velocity is found using the selected fractional order hold or set of $\beta-F R O H$ between sampling instants from the discrete feed velocity. The continuous time of the reference model is obtained as the output of the reference model when a continuous reference force is used as input. Thus, this cost function calculates the area between the continuous-time domain system output response and 
the continuous-time model reference response. The smaller this area is the better continuous-time response will be. Then, the output of the system associated to the lowest value of this cost function will correspond to a signal that better follows the reference signal. In terms of milling processes, this fact implies better accuracy on the work-piece surface and less damage to the tool and machine tool components.

\section{Simulation examples}

In this section, the control schemes introduced above are implemented in the milling system to show the usefulness of the control schemes.

For simulation purposes, a sample workpiece with the profile represented in figure 3 is used, which depicts basic cutting of geometry features with changes in the axial depth of cut. The spindle speed remains constant at $715 \mathrm{rpm}$; the work-piece is made of aluminium 6067 whose specific cutting pressure is assumed to be $K_{c}=1200 \mathrm{~N} / \mathrm{mm}^{2}$. A 4-flute carbide milling tool, fully-immersed and roughing milling operation is taken into consideration in this paper. The following parameters have been chosen for simulation purposes: $\tau_{m}=0.1 s, \tau_{c}=1 / N_{t} S_{s}, r=4$, $\omega_{n}=2.5 / T_{r} \mathrm{rad} / \mathrm{s}, \xi=0.7$ and $S=0.8$. The sampling time is chosen to be inversely proportional to the spindle speed $T=1 / S s$, in this example, $T=60 / 715 \mathrm{~s}$. Also, note that the desired final geometry of the workpiece to be milled involves changes in the axial depth of cut which implies sudden changes in its value, according to the sudden changes assumption. Then the axial deep of cut function $a_{d c}$ in (2) may be time-

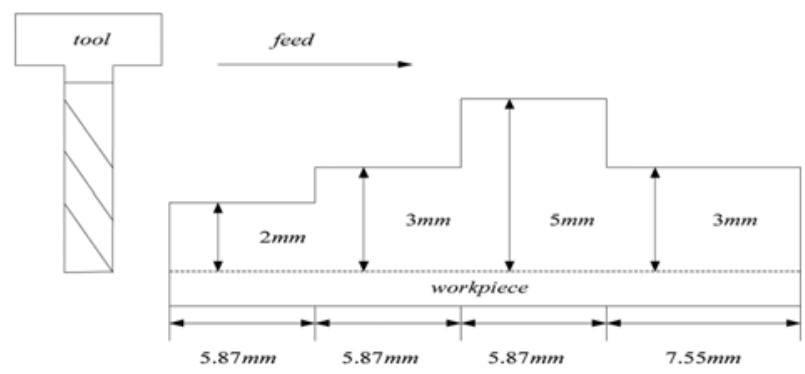

Fig. 3 Workpiece profile to test control algorithms
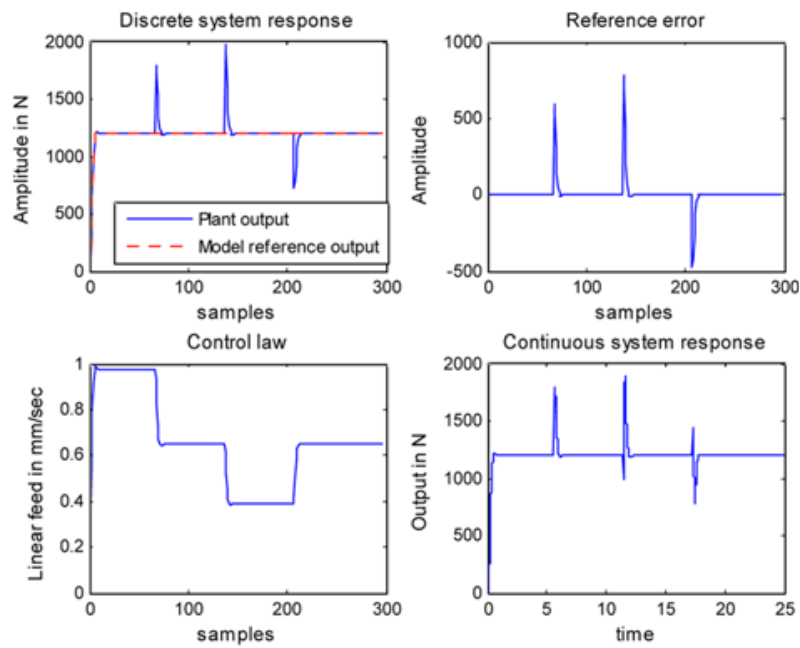

Fig. 4 Responses of the system using a $\mathrm{ZOH}$ varying leading to a potential time-varying system. In particular, the cutting process is assumed to be in this work piecewise constant, admitting sudden changes in the cutting parameters at certain time instants while remaining invariant between changes. This assumption allows us to consider the cutting process to be described by the transfer function (2) with the time interval between changes. On the other hand, it has been taken into account that the control law computes a new feed-rate command value at each sampling interval. Furthermore, it is worth mentioning that the $\mathrm{CNC}$ unit has its own digital position law executed at small time intervals in comparison with the sampling time of the control law, even when high speed milling tool drives are used.

\subsection{Basic model following scheme}

The basic model following scheme depicted in figure 1 is illustrated as simulation outputs. In this sub-section, it is just intended to show the simulation results for fixed gains of the $\beta-F R O H$. As an example, the gain of $\beta=0$ leading to a $Z O H$ and $\beta=-0.3$ has been selected for illustration purposes. Those values have not been selected randomly. They are used as a benchmark to test multi-model schemes.

Thus, figure 4 shows four plots. The first window represents the discrete time force output versus the output of the reference model, the second pictures the error of the system $\left(e=F_{p}-F_{p m}\right)$, the third the control signal and the fourth the continuous time response of the force output.

In comparison, figure 5 pictures the same responses for the case where a fixed gain $-0.3-F R O H$ is programmed.

The reason for plotting those $\beta-F R O H$ is that the $Z O H$ gives us a benchmark of the previously defined cost function performances relative to that used on the shop floor. However, the $-0.3-F R O H$ is plotted to show the potential performance of the $\beta-F R O H$ which can be found, for example in, ${ }^{14}$ where the used $\beta-F R O H$ with negative values of gain gives better performance indexes with respect to $\beta$ equal to zero or positive values.

\subsection{Multi-model schemes}

Two selected plots are used tentatively for the multi-model milling system output performance evaluation. The first one uses the first multi-model scheme which lets the system choose among any of the possible gains, $\beta$. The set of possible gains for evaluating
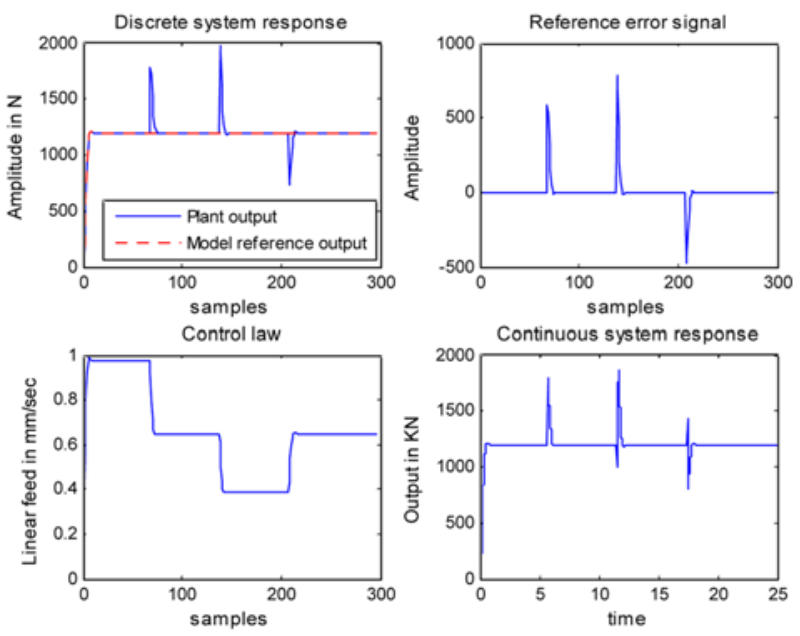

Fig. 5 Responses of the system using a $-0.3-F R O H$ 
the control scheme is selected as $\beta^{(i)}=1-(i-1) / 4$ for $1 \leq i \leq 9$ and the residence time is chosen to be two sampling periods due to low spindle speed machining. For practical purposes, the following parameters in the cost function of the equation (13) has been selected, $\alpha_{k}=0.8, \beta_{k}=0.2, \gamma_{k}=\delta_{k}=0, \forall k$ and $M=4$. Meanwhile, in the second multi-model method the initial value of $\Delta \beta$ is set to 0.2 and $m$ to 2 and $\alpha_{k}=0.8, \gamma_{k}=0.2, \beta_{k}=\delta_{k}=0, \forall k$ and $M=4$ values for the parameters of the cost function has been chosen. The following subsections show the results obtained with the two multimodel schemes presented above.

\subsubsection{Multi-model scheme 1}

Figure 6 depicts discrete-time versus reference model force outputs, signal error $\left(F_{p}^{(l)}-F_{p m}^{(l)}\right)$, control law and continuous time response of the output when the multi-model scheme control is applied.

Meanwhile, figure 7 represents the active value of $\beta$ when it is carrying out the control. The figure plots the value of $\beta$ at each residence time which has been selected to be two periods of time. The switching rule is programmed to stop after thirty period times if the $\beta$ value of the system is not stabilized by itself selecting the value of $\beta$ that the controller has at this moment.

\subsubsection{Multi-model scheme 2}

Figure 8 depicts discrete-time versus reference model force outputs, signal error $\left(F_{p}^{(l)}-F_{p m}^{(l)}\right)$, control law and continuous time response of
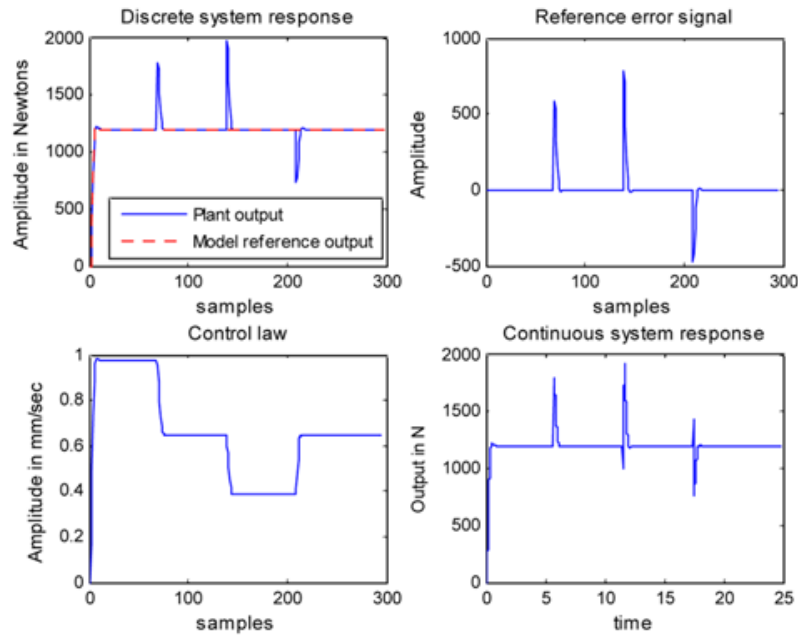

Fig. 6 Responses of the system using multi-model scheme 1

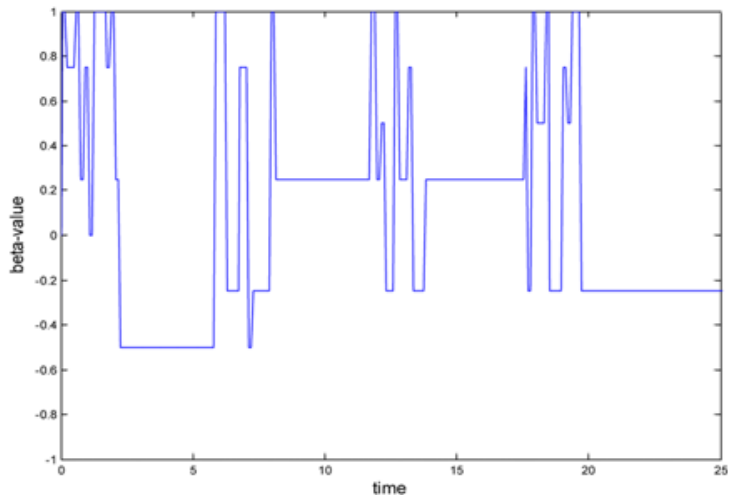

Fig. 7 Active value of with method 1 the output when the multi-model scheme control 2 is applied.

Meanwhile, figure 9 illustrates the active value of $\beta$ when the multimodel scheme 2 is carried out. This plot figures out the values of $\beta$ at each residence time which is selected as two periods. Also, the switching rule is stopped after thirty time periods if the system is not stabilized by itself choosing the value of the gain $\beta$ the control scheme is scheduled at this moment.

\subsection{Analysis of the outputs}

For each synthesized control four output figures are plotted in the group of figures 4, 5, 6 and 8. The first one depicts model reference and plant outputs signals in discrete time; the second one shows the evolution of the tracking error signal $\left(e=F_{p}-F_{p m}\right)$ in the basic model scheme (figures 4 and 5) or $\left(e=F_{p}^{(l)}-F_{p m}^{(l)}\right)$ in the multi-model case (figures 6 and 8); the third figure displays the resultant control signal and, finally, the fourth graphic reveals the continuous time-domain system response of the system. Then, figures 4, 5, 6 and 8 show the outputs, and figure 7 and 9 the on-line active value of $\beta$ selected via switching using (9), for the multi-models methods 1 and 2, respectively.

As it can be appreciated in figures 4, 5, 6 and 8, the steady-state of the resultant force tracks the reference force which is set to $1200 \mathrm{~N}$, except for the response peaks appreciated when the axial depth of cut, and consequently the transfer function, is suddenly altered. Then, a parameter of the system changes abruptly, in this case the axial depth of cut, the model-reference controllers lead to large overshoots, due to
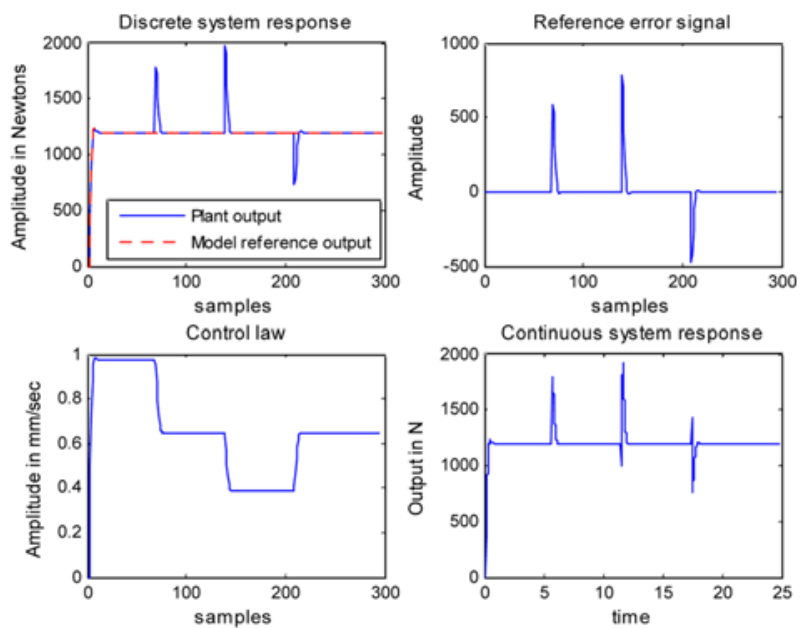

Fig. 8 Responses of the system using multi-model scheme 2

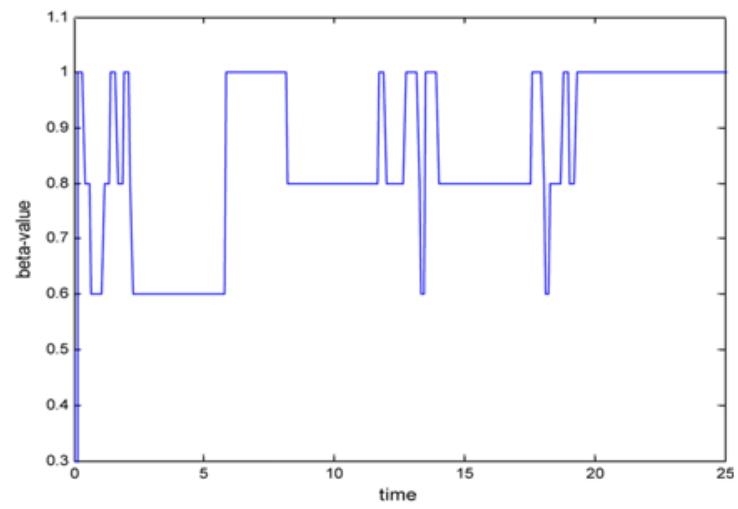

Fig. 9 Active value of $\beta$ with method 2 
the intrinsic structure of the scheme. Thus, if the reference force is selected near the tool breakage limit, the large overshoot would lead to fracture or breakage of the tool. ${ }^{23}$ A solution for this problem is given in ${ }^{24}$ where some 'a priori' information about the workpiece geometry is obtained from the CAD model of the workpiece in order to modify the control command the axial depth of cut changes to minimize the overshoots due to abrupt changes in the axial depth of cut. A drawback could be that the controller cannot respond quickly enough to sudden changes in the cut geometry to eliminate the large spikes in cutting forces. But, it is independent of the multi-model scheme which operates with the various models in parallel disposal so that the computation time due to such a disposal is not significantly increased with respect to the use of a single scheme. Moreover, it is also appreciated that the discrete-time transient response follows exactly the discrete model reference at each sampling instant as a consequence of the perfect knowledge of the plant parameters. Furthermore, the control signal is feasible and smooth, even though when the axial depth of cut varies in every case presented in this paper. Also, note that the value of $\beta$ is independent of the value of the programmed feed rate. Thus, the similitude of the four control signals or programmed feed rates in figures 4, 5, 6, and 8 which are independent of the designed controller. Finally, the continuous-time force response is obtained as the output to the continuous transfer function when it is input the continuous feed velocity control signal using the corresponding $\beta-F R O H$ or the set of the switched $\beta$ parameters. This last output gives information about the true output of the system since the milling system is presented as continuous system.

Multi-model schemes are intended to lead to automatically switch the value of $\beta$ in order to achieve better performance in the output continuous time responses attending to some signal performances through the performance index of the equation (13) and switching rules associated to control schemes 1 and 2 . In the analysis of multi-model scheme 1 it can be observed that the $\beta$-value of the control tends to different values of $\beta$ before reaching the stationary state. At each residence time, the performance index is evaluated and minimized. The switching rule of the multi-model scheme 1 chooses, among the controllers associated to the available parallel models, the one with better performance. This performance, in case of multi-model 1, is related to the output of the closed loop and exhibits fewer jumps in the output, respecting their weighting factors. In the case of multi-model scheme 2, its associated switching rule must be taken into account, as well as the defined performance index that in this case balances the output of the closed loop and cost of the control with their programmed weighting factors. This means that the multi-model scheme does not switch to the controller with better performance output according to the benchmark of equation (14).

\section{Results and discussion}

To test the performance of $\beta-F R O H$ in close-loop represented in figure 1 the cost function explained in equation (14) is used as performance index to evaluate it. Those kinds of performance indexes are perfectly justified as a benchmark to evaluate the output of the control systems. ${ }^{21,22}$ Also, the values of multi-models 1 and 2 are added in this study. Then, figure 10 reveals the results of this study. In blue are; the values of the cost functions of equation (14) ranging for the values of the gain $\beta$ relating to the close loop of figure 1. Also, the values for multi-models 1 and 2 are added as a square and ellipse respectively. It can be observed in this graph for negative values of $\beta$ better performance is achieved in terms of how well the continuous output signal follows the reference signal, which finally is one main objective of the model reference control scheme. The physical meaning for being a negative value of $\beta$ may be that in some types of transients responses associated with rippling in-between samples, it can be proven that the optimal $\beta-F R O H$-gain for signal reconstruction is negative (see, for instance ${ }^{25}$ ). An intuitive explanation can be that sometimes the more relevant response overflows are negative (i.e. underflows) so that the hold gain accommodation can be better performed with negative correcting gains.

The fact the second multi-scheme method gives worse results in this case does not guaranty better performance of the first one. It depends on the case dealt with as it can be checked in. ${ }^{26}$

One potential benefit of using $\beta-F R O H$ can be found when manufacturing un-deformed workpieces profiles, where the characteristics of the $\beta-F R O H$ lead to achieve better surface finish than traditional $\mathrm{ZOH}$ when applying digital controllers. This fact can be intuitively seen in the case the profile of the workpiece varies the axial depth of cut in a ramp or slope form, where $\beta-F R O H$ properties facilitate following the input axial depth of cut variation in comparison with $\mathrm{ZOH}$. This situation is quite common in manufacturing environments. However, this has not been taken into account in this study since the slope of the ramp in the axial depth of cut depends on the control signal or feedrate. To address this, an estimation algorithm can be included in the control scheme, but this is out with the aim of this study.

\section{Conclusions}

In this paper, a discrete-time model following strategy for a known continuous-time milling system has been developed. Two different schemes have been developed, both basic and multi-model schemes. The novelty of the basic control relies on the use of a $\beta-F R O H$ in the

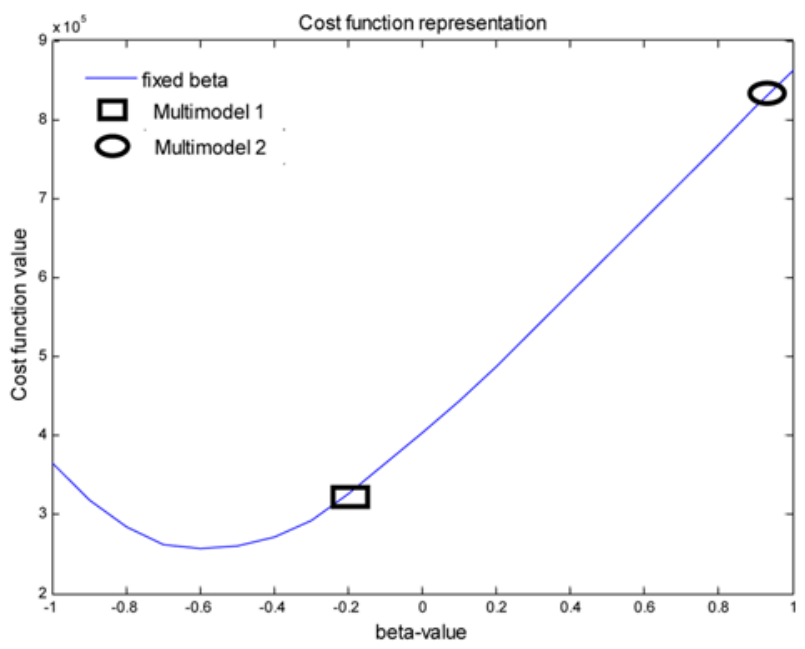

Fig. 10 Cost function vs. $\beta$-value 
discretization process instead of the usual $\mathrm{ZOH}$ appearing in the manufacturing literature. The introduction of an additional "degree of freedom" provided by the correcting gain of the $\beta-F R O H$ allows the designer to improve transient responses, the properties of the zeros of the transfer function and the inter-sample behavior of the continuous time close loop system by adequate selecting of its value; this result has been borne out by the simulation results. Furthermore, the multi-model scheme is designed to find at each multiple residence time the value of the gain $\beta$ which leads to a balance among performance of the closedloop, the jump in the output associated to a switching between controls, the cost of the control and the output signal error, picturing the performance index as cost function. Two methods have been presented for this purpose. The first one selects the current value of the gain among a fixed set of possible predetermined values. The second one updates $\beta$ only to a close value of the active one, avoiding bad transient in switching instants due to big changes in the $\beta$-FROH gain. This kind of multi-parallel control scheme is typically used for modifying the closed-loop structured, as for this study, or to propose solutions to systems which can work in different states governed by different equations, as for the general milling problem.

Moreover, an auxiliary cost function which measures the continuous-time response performance of the output force is introduced. This cost function gives an approximate area between the continuous-time output to the system and the continuous-time output of the desired output model reference. The simulations shows that an appropriate choice of fixed value of $\beta$ leads to a better continuous-time response than when just a $\mathrm{ZOH}$ is used. Also, the multi-model-scheme 1 outputs better continuous-time response than the $\mathrm{ZOH}$ closed loop. However, the multi-model scheme 2 is not able to achieve the same performance as $\mathrm{ZOH}$ basic control scheme in respect of the continuoustime response evaluation cost function.

Another potential benefit of using $\beta-F R O H$ instead of $Z O H$ resides in more reliable reconstruction of the continuous output, especially, when ramp or free form signals input the system. Also, the parallel model scheme, in spite of using it in this paper as enhancement of single working point, can be used in to design a control scheme for milling in different states.

Finally, the $\beta-F R O H$ device can be implemented by means of $Z O H$, which makes this approach readily feasible for implementing in manufacturing industry.

\section{ACKNOWLEDGEMENTS}

The authors gratefully acknowledge the UK's Engineering and Physical Science Research Council (EPSRC) funding of the EPSRC Centre for Innovative Manufacturing in Advance Metrology (Grant Ref: EP/I033424/1).

\section{REFERENCES}

1. Ko, J. H. and Shaw, K. C., "Chatter prediction based on frequency domain solution in CNC pocket milling," Int. J. Precis. Eng. Manuf., Vol. 10, No. 4, pp. 19-25, 2009.
2. Ko, J. H. and Tan, S. W., "Chatter marks reduction in meso-scale milling through ultrasonic vibration assistance parallel to to tooling's axis,” Int. J. Precis. Eng. Manuf., Vol. 14, No. 1, pp. 17-22, 2013.

3. Ko, J. H., "Plunge milling force model using instantaneous cutting force coefficients," Int. J. Precis. Eng. Manuf., Vol. 7, No. 3, pp. 813, 2006.

4. Kamguem, R., Tahan, S. A., and Songmene, V., "Evaluation of machined part surface roughness using image texture gradient factor,” Int. J. Precis. Eng. Manuf., Vol. 14, No. 2, pp. 183-190, 2013.

5. Pa, N. M. N., Sarhan, A. A. D., and Shukor, M. H. A., "Optimizing the cutting parameters for better surface quality in $2.5 \mathrm{D}$ cutting utilizing titanium coated carbide ball end mill,” Int. J. Precis. Eng. Manuf., Vol. 13, No. 12, pp. 2097-2102, 2012.

6. Masory, O. and Koren, Y., "Adaptive control system for turning," Annals of the CIRP, Vol. 29, No. 1, pp. 281-289, 1980.

7. Masory, O. and Koren, Y., "Adaptive control with process estimation," Annals of the CIRP, Vol. 30, No. 1, pp. 373-376, 1981.

8. Tomizuka, M., Oh, J. H., and Dornfeld, D. A., "Model reference adaptive control of milling process," Control of Manufacturing Processes and Robotic Systems, ASME Annual Meeting, pp. 55-63, 1983.

9. Landerbaugh, L. K. and Ulsoy, G., "Model reference adaptive control in milling,” J. of Eng. for Ind., Vol. 111, pp. 13-21, 1989.

10. Altintas, Y., "Manufacturing Automation," Cambridge, 2012.

11. Landers, R. G., Ulsoy, A. G., and Ma, Y. H., "A comparison of model based machining force control approaches," Int. J. of Machine Tools \& Manuf., Vol. 44, No. 7-8, pp. 733-748, 2004.

12. Liu, Y. and Wang, C., "Neural network based adaptive control and optimization in milling processes," Int. J. Adv. Manuf. Tech., Vol. 15, pp. 791-795, 1999

13. Zuperl, U., Cus, F., and Milfelner, M., "Fuzzy control strategy for an adaptive force control in end-milling," J. of Mat. Tech., Vol. 164165, pp. 1472-1478, 2005

14. Denkena, B. and Floter, F., "Adaptive cutting force control on a milling machine with hybrid axis configuration," Proc. of CIRP, pp. 109-114, 2012.

15. Cus, F. and Zuperl, U., "Model reference-based machining force and surface roughness control," J. of Achievements in Mat. and Manuf. Eng., Vol. 29, No. 2, pp. 115-122, 2008.

16. De la Sen, M., "About Optimal Fractional Hold Circuits for Intersample Output Reconstruction in Sampled-data Systems," Sensors, Vol. 7, pp. 3146-3155, 2007.

17. Barcena, R., De la Sen, M., and Sagastabeitia, I., "Improving the stability properties of the zeros of sampled systems with fractional order holds," IEE Proc.-Control Theory \& Applic., Vol. 147, No. 4, pp. 456-464, 2000. 
18. Narendra, K. S. and Balakrishnan, J., "Improving transient response of adaptive control systems using multiple models and switching," IEEE Trans. on Aut. Cont., Vol. 39, No. 9, pp. 1861-1866, 1994.

19. Ishitobi, M., "Stability of zeros of sampled systems with fractional order hold," IEE Proc. Control Theory \& Applications, Vol. 143, No. 3, pp. 296-300, 1993.

20. Astrom, K. J., Hanganger, P., and Sternby, J., "Zeros of sampled systems," Automatica, Vol. 20, No. 1, pp. 31-38, 1984.

21. Ibeas, A., De la Sen, M., and Alonso-Quesada, S., "Stable multiestimation model for single-input/output discrete adaptive control systems,” Int. J. of Syst. Sci., Vol. 35, No. 8, pp. 479-501, 2004.

22. Ibeas, A. and De la Sen, M., "Robustly stable adaptive control of a tandem of master-slave robotic manipulators with force reflexion by using a multi-estimation scheme," IEEE Trans. on Syst. Man \& Cybern., Part B: Cybernetics, Vol. 36, No. 5, pp. 1162-1179, 2006.

23. Altintas, Y., "Prediction of cutting forces and tool breakage in milling from feed drive current measurements," J. of Eng. for Industry, Vol. 114, pp. 386-392, 1992.

24. Spence, A. and Altintas, Y., "CAD assisted adaptive control for milling," Journal of Dynamic Systems Measurement and ControlTransactions of the ASME, Vol. 113, No. 3, pp. 444-450, 1991.

25. De la Sen, M., Baranbones, O., and Garrido, A. J., "Design of fractional circuits for output reconstruction in discretized systems," IEEE/ASME Inter. Conf. on Adv. Intell. Mechat., pp. 668-672, 2009.

26. Bilbao-Guillerna, A., De la Sen, M., and Alonso-Quesada, S., "Online supervised adjustment of the correcting gains of fractional order holds," Proc, of ICINCO, pp. 266-272, 2005. 